\title{
Preditores de mudanças nos regimes terapêuticos para o tratamento de Aids em crianças
}

\author{
Predictors of changes in drug regimens for treating AIDS in children
}

\author{
Cristina Ribeiro Macedo ${ }^{4}$, Diana de Oliveira Frauches ${ }^{1,4}$, Laylla Ribeiro Macedo ${ }^{3}$, \\ Sandra Fagundes Moreira-Silva ${ }^{1,4}$, Priscila Rocha de Araújo Nader ${ }^{4}$, \\ Ana Paula Neves Burian Lima ${ }^{1}$ e Ethel Leonor Nóia Maciel $^{2,3}$
}

\begin{abstract}
RESUMO
Identificar as causas de mudanças de esquemas terapêuticos no tratamento de Aids em crianças contaminadas por transmissão vertical. Estudo casocontrole, sendo o grupo controle constituído de 49 crianças que não efetuaram troca de esquema antirretroviral, e o grupo caso, de 62 crianças que já efetuaram troca de janeiro de 2000 a dezembro de 2005. Foram utilizados dados do prontuário e roteiro de entrevista semi-estruturada. A análise dos dados foi pelo programa SPSS, usado teste qui-quadrado e regressão logística. As principais causas para mudança do tratamento foram: piora virológica (48,4\%), imunológica (46,6\%) e clínica (35,5\%) dos pacientes. 0 ajuste dos dados através de análise da regressão logística demonstrou que a intolerância medicamentosa foi a variável que mais contribui para a mudança da medicação (OR austado $_{7}: 99,94 ;$ IC $\left.95 \%: 6,28-1034,55\right)$ A adesão não foi apontada como responsável pela troca de tratamento antirretroviral, esse fato foi atribuído à organização do serviço e a atuação da equipe interdisciplinar.
\end{abstract}

Palavras-chaves: Aids. Crianças. Tratamento. Falha. Adesão

\begin{abstract}
To identify the causes of changes in therapeutic regimens for treating AIDS in children infected through vertical transmission. This was a case-control study in which the control group consisted of 49 children who had not had any changes to their antiretroviral regimen and the case group consisted of 62 children who had had changes between January 2000 and December 2005. Data from the patients' medical files and a semistructured interview were used. The data analysis was carried out using the SPSS software, and the chi-square test and logistic regression were applied. The main causes of changes in treatment were: increased viral load (48.4\%), poor immunological response (46.6\%) and clinical worsening (35.5\%) of the patients. The adjustment of the data through logistic regression analysis showed that drug intolerance was the variable that contributed most to the change in medication ( ${ }_{\text {adiusted }}$ OR: 79.94; 95\% CI: 6.28 -1034.55). Adherence was not shown to be responsible for changes in the anti-retroviral therapy, and this was attributable to the organization of the service and actions of the interdisciplinary team.
\end{abstract}

Key-words: Aids. Children. Treatment. Failure. Adherence.

A instituição criteriosa de terapia antirretroviral, no tratamento de Aids é conduta importante, no sentido de proporcionar maior sobrevida aos pacientes ${ }^{6}$.

No entanto, em crianças cuja transmissão vertical foi diagnosticada um dos grandes problemas apontados é a resposta sustentada da terapia, relacionada à potência, tolerância e possibilidade de boa qualidade de vida durante o tratamento da Aids ${ }^{7}$.

1. Serviço de Infectologia, Hospital Infantil Nossa Senhora da Glória, Vitória, ES. 2. Núcleo de Doenças Infecciosas, Universidade Federal do Espírito Santo, Vitória, ES. 3. Universidade Federal do Espírito Santo, 4. Escola Superior de Ciências da Santa Casa de Misericórdia de Vitória, Vitória, ES.

Endereço para correspondência: Dr ${ }^{\mathrm{a}}$ Cristina Ribeiro Macedo. Serviço de Infectologia do Hospital Infantil Nossa Senhora da Glória. Alameda Mary Ubirajara 205, 29056-030 Vitória, ES.

Tel: 5527 3337-3927/9999-5033.

e-mail: cristinarmacedo@gmail.com

Recebido para publicação em 13/04/2009

Aceito em 05/11/2009
Alguns obstáculos são frequentemente responsáveis pela diminuição da resposta sustentada ao tratamento: a condição clínica do paciente, a de piora imunológica, o aumento da carga viral, que em conjunto ou individualmente são responsáveis pela determinação de falha terapêutica de esquema antirretroviral ${ }^{1}$.

A intolerância aos antirretrovirais, outra determinante para troca de esquema, é considerada quando o paciente mostra impossibilidade absoluta de uso do medicamento, apresentando efeitos colaterais incompatíveis com a qualidade de vida ${ }^{2}$. Além disto, a falha terapêutica, também pode estar relacionada com a adesão ao tratamento e essa possibilidade sempre deve ser investigada ${ }^{10}$.

Os regimes terapêuticos disponibilizados pelo Ministério da Saúde para crianças em tratamento de Aids podem ser agrupados em três classes de medicamentos: $1^{\text {a }}$ ) Inibidores de fusão (efurvitida: não estava disponível à época do estudo). $2^{\text {a }}$ ) Inibidores da transcriptase reversa (análogo de nucleosídeo: estavudina, lamivudina, tenofovir e zidovudina; não-análogo do nucleosídeo: efavirenz e nevirapina). $3^{\text {a }}$ ) Inibidores da protease (amprenavir, atazanavir, indinavir, lopinavir/ritonavir, 
nelfinavir, ritonavir, saquinavir). Cada uma destas classes oferece várias opções de fármacos, sendo passível de em caso de uso de algum destes, o paciente desenvolver resistência cruzada a medicamentos da mesma classe ${ }^{1}$.

Trocas de esquema de tratamento de Aids podem implicar em redução de opções possíveis em uma nova terapia, pela resistência cruzada que poderá se desenvolver a fármacos da mesma classe. Sabe-se que apesar de haver um número aparentemente grande de drogas disponíveis, há sempre que se pensar em limitações técnicas impostas por estas ${ }^{25}$. Neste contexto, conhecer os determinantes da troca é de fundamental importância para se prever possíveis falhas terapêuticas antes que ela ocorra. O objetivo deste estudo foi identificar as causas de mudanças de esquemas terapêuticos no tratamento de Aids em crianças contaminadas por transmissão vertical, atendidas no Hospital Infantil Nossa Senhora da Glória (HINSG).

\section{MATERIAL E MÉTODOS}

Este foi um estudo caso-controle realizado no município de Vitória através da aplicação de um questionário estruturado para avaliar fatores preditores de troca de tratamento antirretroviral.

o local da coleta de dados foi o Serviço de Infectologia do Hospital Infantil Nossa Senhora da Glória referência estadual para 0 atendimento de crianças com HIV/AIDS.

Um instrumento contendo perguntas fechadas foi aplicado ao cuidador das crianças, sujeitos do estudo, sempre após a assinatura do termo de consentimento pós-informação relativo a hábitos que pudessem ter interferido na troca de medicamento. Duas pesquisadoras treinadas fizeram as entrevistas. Após esta avaliação a equipe do estudo coletou dados no prontuário clinico através de formulário estruturado contendo: dados sociodemográficos, resultados de exames laboratoriais, data do diagnóstico, início da terapia, tempo de utilização de cada esquema, motivo da troca da medicação, bem como, informações sobre efeitos adversos. Estabeleceu-se por intolerância, a existência de efeitos colaterais incompatíveis com boa qualidade de vida e que determinaram suspensão do esquema antirretroviral.

Alem disso, a adesão medicamentosa foi definida como: manutenção do mesmo horário na administração dos medicamentos, aceitação da criança e assiduidade da retirada da farmácia. Outros fatores foram avaliados para reforçar a aferição da adesão, tais como: utilização de estratégias para maior aceitação do medicamento e entendimento do cuidador em relação à receita médica, além disso, os pacientes que apresentaram três faltas ou mais ao serviço, no período de seis meses, foram considerados não aderentes, independente das outras variáveis.

Entre janeiro de 2000 e janeiro de 2005, período da realização deste estudo, 230 pacientes encontravam-se em acompanhamento no serviço de Serviço de Infectologia do Hospital HINSG.

Para a seleção dos casos, foram observados os seguintes critérios: estar em tratamento e ter efetuado pelo menos uma troca de esquema terapêutico durante o período do estudo.
Para a seleção dos controles, foram observados os seguintes critérios: estar no primeiro tratamento sem ter tido troca de esquema terapêutico.

Das crianças analisadas, 111 estavam em uso de antirretroviral ${ }^{7}$. Para avaliação e categorização do desfecho (troca do medicamento), foram analisados todos os prontuários da farmácia. A partir desta análise, 62 crianças foram categorizadas como caso: realizaram pelo menos uma troca do antirretroviral no período do estudo e 49 como controles, ou seja, no período estudado haviam permanecido com o mesmo esquema terapêutico.

As informações coletadas foram codificadas e armazenadas em um banco de dados, usando o programa Statistic Package for the Social Science (SPSS) versão 16.0 para Windows. Na avaliação comparativa das variáveis estudadas, empregou-se o teste quiquadrado $(\chi 2)$ e o teste exato de Fisher quando apropriado, ambos de forma bicaudal e com nível de significância de 0,05 . A associação entre a variável dependente (troca de esquema) e as variáveis explicativas foram avaliadas pela razão de chances (odds ratio) e seus respectivos intervalos de confiança de $95 \%$. Na modelagem estatística, selecionaram-se as variáveis cujos valores de p (nível de significância descritivo do teste) foram menores ou iguais a 0,20 na análise bruta. A seguir, essas variáveis foram introduzidas no modelo de regressão logística por meio do procedimento stepwise. Foram mantidas no modelo as variáveis que permaneceram significativas depois do ajuste pelas outras variáveis.

0 projeto foi aprovado pelo Comitê de Ética e Pesquisa do HINSG, em 7 de abril de 2005. Os cuidadores (responsáveis legais pela criança) assinaram um Termo de Consentimento Livre e Esclarecido.

\section{RESULTADOS}

Observou-se que a distribuição segundo sexo foi semelhante nos dois grupos. Na distribuição segundo idade, observou-se um número proporcional de crianças no grupo que efetuou troca de esquemas, sendo na faixa etária inferior a dez anos de idade $48(53,9 \%)$ e acima de dez anos de idade 14 (53,6\%), no grupo controle houve um predomínio de crianças abaixo de dez anos 41 (46,1\%) e acima de dez anos 8 (36,4\%). Quanto à procedência, verificou-se maior número de pacientes procedentes da Grande Vitória, sendo no grupo de casos encontrados 46 (74,2\%) crianças e no grupo controle 39 (79,9\%) crianças (Tabela 1).

Com relação à idade de início da terapia, observou-se que $29(72,5 \%)$ das crianças cujo tratamento iniciou-se acima de cinco anos apresentaram pelo menos uma troca no regime terapêutico inicial em comparação com $33(46,5 \%)$ das que se trataram antes de desta faixa etária (OR: 3,03; IC95\%: 1,23-7,76).

Considerando-se os critérios para avaliar troca de esquema por resistência ou falha terapêutica, observamos que alguns pacientes atendiam a mais de um critério. A piora virológica apresentou-se em 37 (59,6\%) dos casos, em contrapartida 25 (40,3\%) crianças deste grupo não apresentaram piora clínica, a piora imunológica em $39(62,9 \%)$ dos casos e $23(37,1 \%)$ não apresentaram, a piora clínica em 20 (32,2\%) e a intolerância em 
TABELA 1

Distribuição de crianças e OR com seus intervalos de confiança, segundo grupos relacionados (n⿳o =111). Dados coletados de abril a junho de 2005. HINSG/Vitória, ES.

\begin{tabular}{|c|c|c|c|c|c|c|}
\hline \multirow[b]{2}{*}{ Variáveis } & \multicolumn{2}{|c|}{ Caso } & \multicolumn{2}{|c|}{ Controle } & \multirow[b]{2}{*}{ OR(IC 95\%) } & \multirow[b]{2}{*}{ p-valor } \\
\hline & $\mathrm{n}^{0}$ & $\%$ & $\mathrm{n}^{0}$ & $\%$ & & \\
\hline \multicolumn{7}{|l|}{ Sexo } \\
\hline feminino & 28 & 50 & 28 & 50 & $0,62(0,27-1,41)$ & 0,287 \\
\hline masculino & 34 & 61,8 & 21 & 38,2 & & \\
\hline \multicolumn{7}{|l|}{ Faixa etária (anos) } \\
\hline entre 0 e 10 & 48 & 53,9 & 41 & 46,1 & $0,67(0,23-1,23)$ & 0,561 \\
\hline acima de 10 & 14 & 53,6 & 8 & 36,4 & & \\
\hline \multicolumn{7}{|l|}{ Procedência } \\
\hline grande Vitória & 46 & 54,1 & 39 & 45,9 & $0,74(0,27-1,97)$ & 0,659 \\
\hline interior/outros estados & 16 & 61,5 & 10 & 38,5 & & \\
\hline \multicolumn{7}{|c|}{ Idade no início da terapia (anos) } \\
\hline até 5 & 33 & 46,5 & 38 & 53,5 & $3,03(1,23-7,76)$ & 0,008 \\
\hline acima de 5 & 29 & 72,5 & 11 & 27,5 & & \\
\hline
\end{tabular}

OR: odds ratio, IC 95\%: intervalo de confiança.

$10(16,1 \%)$ dos casos estudados, sendo que respectivamente $42(67,8 \%)$ e $52(83,9 \%)$ não apresentaram.

Algumas crianças no grupo dos controles também apresentaram piora imunológica, virológica e clínica e estes dados foram avaliados na Tabela 2.

Observou-se uma chance de troca por piora imunológica 12.5 vezes maior no grupo caso em relação ao grupo controle. A piora clínica foi a segunda causa de mudança do esquema com uma chance de 5,36 (1,55-20,3). Por outro lado, a intolerância a menor chance de mudança de tratamento. Foi observado nos prontuários que a maioria dos efeitos colaterais apresentados era passível de resolução, e ocorreu principalmente no início da terapia, com melhora em curto período de tempo.

Dentre os parâmetros utilizados para avaliar a adesão, observou-se que a assiduidade no grupo caso foi bastante satisfatória $39(62,9 \%)$ das crianças não faltam ao serviço e $23(37,1 \%)$ tiveram mais de duas faltas em seis meses. Sendo que no grupo controle houve uma diferença maior no grupo que não faltam as consultas, $35(71,4 \%)$ e $14(28,6 \%)$ tiveram mais de duas faltas em seis meses, não havendo diferenças ente os grupos estudados OR: 0,7 (IC: 0,27-1,63). Quanto à manutenção dos horários de medicamentos no grupo caso, observou-se que $55(88,7 \%)$ das crianças compatibiliza os horários e apenas $7(11,2 \%)$ adianta ou atrasa. Muitos dos que trocaram de tratamento antirretroviral souberam informar o nome dos medicamentos $40(64,5 \%)$ e $22(35,5 \%)$ não o fizeram. Em relação à leitura da receita dos medicamentos, 54 (87\%) dos entrevistados, do grupo caso, responderam que é possível fazer e apenas 8 (13\%) no mesmo grupo não o fazem, sendo que para o ,mesmo parâmetro no grupo controle 39 (79,6\%) disseram sim e 10 (20,4\%) não. 0 medicamento era retirado regularmente da farmácia no grupo caso por $43(69,4 \%)$ sendo que 19 (30,6\%) não o fazem, no grupo controle o fato ocorre 37 (75\%) e 12 (25\%), respectivamente.

Em relação às estratégias utilizadas para a adesão ao tratamento, as respostas estratificadas por grupo de estudo estão demonstradas na Tabela 3. Destaca-se que não houve diferenças significativas entre os grupos, que pudesse justificar a falta de adesão como responsável pela troca de terapia antirretroviral.

TABELA 2

Distribuição dos pacientes segundo o motivo de troca de tratamento antirretroviral; piora virológica, imunológica, clínica e intolerância $\left(\mathrm{n}^{\mathrm{0}}=111\right.$ ). Dados coletados de abril a junho de 2005. HINSG/Vitória, ES

\begin{tabular}{|c|c|c|c|c|c|c|}
\hline \multirow[b]{2}{*}{ Piora virlógica } & \multicolumn{2}{|c|}{ Caso } & \multicolumn{2}{|c|}{ Controle } & \multirow[b]{2}{*}{ OR(IC 95\%) } & \multirow[b]{2}{*}{ p-valor } \\
\hline & $\mathrm{n}^{0}$ & $\%$ & $\mathrm{n}^{0}$ & $\%$ & & \\
\hline $\operatorname{sim}$ & 37 & 59,6 & 18 & 36,7 & $2,55(1,10-5,95)$ & 0,016 \\
\hline não & 25 & 40,3 & 31 & 63,3 & & \\
\hline \multicolumn{7}{|c|}{ Piora imunológica } \\
\hline sim & 39 & 62,9 & 6 & 12,2 & $12,5(4,2-37,7)$ & 0,001 \\
\hline não & 23 & 37,1 & 43 & 87,8 & & \\
\hline \multicolumn{7}{|l|}{ Piora clínica } \\
\hline $\operatorname{sim}$ & 20 & 32,2 & 4 & 8,2 & $5,36(1,62-23,01) *$ & 0,002 \\
\hline não & 42 & 67,8 & 45 & 91,8 & & \\
\hline \multicolumn{7}{|l|}{ Intolerância } \\
\hline $\operatorname{sim}$ & 10 & 16,1 & 1 & 2,0 & $9,23(1,2-408,7)^{*}$ & 0,013 \\
\hline não & 52 & 83,9 & 48 & 98 & & \\
\hline
\end{tabular}

OR: odds ratio, IC 95\%: intervalo de confiança.

*Usado o teste exato de Fisher que melhor se adéqua a situação. 
TABELA 3

Distribuição dos pacientes segundo a adesão ao tratamento antirretroviral, utilizando os seguintes parâmetros: faltas, estratégias na administração e manutenção de horários e outros ( $\left.n^{0}=111\right)$. Dados coletados de abril a junho de 2005. HINSG/Vitória, ES.

\begin{tabular}{|c|c|c|c|c|c|c|}
\hline \multirow[b]{2}{*}{ Variáveis } & \multicolumn{2}{|c|}{ Caso } & \multicolumn{2}{|c|}{ Controle } & \multirow[b]{2}{*}{ OR (IC 95\%) } & \multirow[b]{2}{*}{ p-valor } \\
\hline & $\mathrm{n}^{\underline{0}}$ & $\%$ & $\mathrm{n}^{\mathrm{o}}$ & $\%$ & & \\
\hline \multicolumn{7}{|l|}{ Assiduidade } \\
\hline não falta ou raramente falta & 39 & 62,9 & 35 & 71,4 & $0,68(0,28-1,63)$ & 0,827 \\
\hline mais de 2 faltas em 6 meses & 23 & 37,1 & 14 & 28,6 & & \\
\hline \multicolumn{7}{|l|}{ Compatibiliza os horários } \\
\hline mantém o horário & 55 & 88,7 & 38 & 77,5 & $2,27(0,73-7,24)$ & 0,113 \\
\hline adianta ou atrasa & & 7 & 11,2 & 11 & 22,5 & \\
\hline \multicolumn{7}{|l|}{ Estratégia } \\
\hline utiliza & 31 & 50,0 & 29 & 59,2 & $0,69(0,30-) 1,18$ & 0,439 \\
\hline não utiliza & & 31 & 50,0 & 20 & 40,8 & \\
\hline \multicolumn{7}{|l|}{ Diz o nome do medicamento } \\
\hline $\operatorname{sim}$ & 40 & 64,5 & 34 & 69,3 & $0,80(0,33-1,57)$ & 0,35 \\
\hline não & & 22 & 35,5 & 15 & 30,7 & \\
\hline \multicolumn{7}{|l|}{ Consegue ler a receita } \\
\hline $\operatorname{sim}$ & 54 & 87 & 39 & 79.6 & $1,73(0,56-1,37)$ & 0,420 \\
\hline não & & 8 & 13 & 10 & 20,4 & \\
\hline \multicolumn{7}{|c|}{ Retirada regular do medicamento } \\
\hline $\operatorname{sim}$ & 43 & 69,4 & 37 & 75 & $0,73(0,55,52$ & 0,613 \\
\hline não & & 19 & 30,6 & 12 & 25 & \\
\hline
\end{tabular}

OR: odds ratio, IC 95\%: intervalo de confiança

Na Tabela 4, está representado o ajuste dos dados através de análise da regressão logística entre a troca de medicação relacionado as outras variáveis selecionadas. Ressalta-se na análise que a intolerância medicamentosa foi a variável que mais contribui para a mudança da medicação $\left(\mathrm{OR}_{\text {ajustado }}: 79,94\right.$; IC95\%:6,28-1034,55) seguida da piora do quadro clínico do paciente $\left(\mathrm{OR}_{\text {ajustado }}: 10,55 ; \mathrm{IC} 95 \%: 2,19-53,34\right)$. Chama a atenção as duas variáveis que se mostraram fator de proteção para a troca de medicação que foram: a idade maior de cinco anos no início da terapia e as crianças cujos horários de medicação foram mantidos rigorosamente pelos cuidadores durante o tratamento.

\section{TABELA 4}

Análise das variáveis estudados ajustadas através da análise de regressão logística em relação a troca de medicação $\left(n^{0}=111\right)$. Dados coletados de abril a junho de 2005. HINSG/Vitória, ES.

\begin{tabular}{lclc}
\hline Variáveis & OR ajustado* & IC $(95 \%)$ & p-valor \\
\hline Piora virológica & 1,45 & $0,80-7,80$ & 0,12 \\
Piora imunológica & 4,47 & $2,16-24,31$ & 0,001 \\
Idade no inico da terapia & 0,30 & $0,09-0,98$ & 0,048 \\
Piora clinica & 10,55 & $2,19-53,34$ & 0,004 \\
Intolerância & 79,94 & $6,28-1034,55$ & 0,001 \\
Mantém horários da medicação & 0,12 & $0,027-0,53$ & 0,005 \\
\hline
\end{tabular}

*odds ratio ajustado para todas as demais variáveis.

\section{DISCUSSÃO}

A escolha do esquema antirretroviral a ser utilizado em pacientes virgens de tratamento é de grande importância. Na avaliação, dos resultados, a partir do objetivo proposto, que foi identificar as causas de mudança de esquemas terapêuticos no tratamento de Aids de crianças contaminadas por transmissão vertical, atendidas no Serviço de Infectologia do HINSG, observouse que o principal fator foi a falha de tratamento pela intolerância medicamentosa e piora clinica. Em 30 (57,6\%) casos, as trocas ocorreram após 24 meses de tratamento, semelhando ao observado por D'armino e cols 5 , sendo que a literatura aponta, que em adultos, $50 \%$ da troca de esquema antirretroviral, ocorre no primeiro ano de tratamento ${ }^{15}$.

No Brasil, possuem poucos trabalhos relacionados à associação de fatores que determinam mudança de esquemas terapêuticos em crianças, mas algumas publicações internacionais discutem o tema, alguns autores apontam a terapia highly active antirretroviral treatment active antirretroviral combination therapy (HAART) como um bom preditor para manutenção de tratamento por período prolongado ${ }^{31725}$, especialmente e em pacientes virgens de tratamento ${ }^{5}$, no presente estudo observa-se algumas crianças em uso de antirretroviral em um período anterior ao uso de esquemas potentes o que, possivelmente contribui para trocas de esquemas de tratamento.

A terapia dupla com inibidores da transcriptase reversa em faixas etárias jovens podem determinar trocas precoces de medicamentos e hoje se procura instituir terapias medicamentosas potentes em pacientes virgens de tratamento ${ }^{19}$, fato que não foi comprovado no estudo, pois a piora clínica e idade de início da terapia não foram determinantes de troca, muitos dos pacientes em estudo encontravam-se em tratamento por muitos anos mantendo terapia dupla.

Observa-se que a descontinuidade de tratamento em um prazo de acima de sete dias pode determinar um aumento de carga viral, se a interrupção de tratamento se prolongar poderá 
ocorrer a redução de contagens de células $\mathrm{CD}^{4} \mathrm{e}$ interferência na resistência viral ${ }^{13}{ }^{16}$. Interrupções de terapia programadas devem ser efetuadas com rigoroso acompanhamento clínico e laboratorial ${ }^{4}$.

A ocorrência de efeitos colaterais incompatíveis com uma adequada qualidade de vida, têm sido observado com frequência em alguns estudos ${ }^{1720}$. D' Armino e cols ${ }^{3} \mathrm{em}$ um estudo para investigar as causas de mudança em esquemas de tratamento antirretroviral ${ }^{5}$ em indivíduos italianos soropositivos, identifica como responsáveis pela troca de tratamento fatores como: toxicidade (21,1\%), falência $(5,1 \%)$, não adesão $(7,1 \%)$ e outros $(36,2 \%)$, o que corrobora com o presente estudo que considera a intolerância uma importante causa de alteração da terapia medicamentosa, quando avaliamos o odds ratio (OR) ajustado de 79,94 com um intervalo de confiança (IC) de 6,28-1034,55.

Outra manifestação importante observado são os efeitos gastrointestinais como: náuseas e vômitos que interferem definitivamente no ritmo de vida dos indivíduos, principalmente nos primeiros três meses da terapia HAART ${ }^{18}$, reforçando a idéia do estudo de que a intolerância é um importante determinante de troca.

Estudos tipo caso-controle são em geral criticados por serem suscetíveis a vieses de seleção e informação. A esses problemas metodológicos foram adicionadas estratégias que permitiram sua minimização tais como: casos e controles provenientes da mesma base populacional, informações coletadas dos cuidadores foram contra checadas através do prontuário e de anotações nos registros da farmácia. Além disto, todos os exames laboratoriais foram avaliados no sentido de estabelecer a confiabilidade destes resultados.

Não foi possível relacionar mudanças dos esquemas terapêuticos com a adesão ao tratamento de Aids. Acredita-se que algumas características do serviço estudado concorreram para que a adesão ao tratamento fosse satisfatória, não representando importante causa de falha terapêutica. Ressalta-se ainda, as dificuldades em aferir a adesão. Neste estudo, utilizou-se cinco critérios diferentes, pois conforme apontado em alguns estudos, quanto mais diferentes métodos forem utilizados, menor a vulnerabilidade em sofrerem influência de fatores externos que alterem 0 resultado ${ }^{811}{ }^{12}$. É clara a associação entre adesão e supressão viral ${ }^{22}$.

Um estudo desenvolvido por Simoni e cols ${ }^{21}$, em Porto Alegre, Rio Grande do Sul, verificou-se a não adesão ao tratamento de Aids em crianças foi de $49,5 \%$ acima da estimada ${ }^{21}$, dificultando a supressão viral, no presente estudo a adesão foi considerada satisfatória nos dois grupos de estudo, não determinando causa de mudança de tratamento.

Em uma recente metanálise de artigos publicados por programas que atendem pacientes HIV pediátricos, os países em desenvolvimento apresentaram uma adesão superior a $75 \%$ enquanto os estudos realizados em paises desenvolvidos apresentaram uma adesão inferior a 75\%, o fato acima estaria relacionado a fatores familiares estruturais, padrão socioeconômico e o regime utilizado, o que reforça a idéia de conhecer estes fatores para se estabelecer estratégias facilitadoras de adesã $0^{24}$. Outro estudo demonstrou que 0 aconselhamento de suporte para pacientes em uso de antirretrovirais foi fundamental para a melhora na aderência ao tratamento?

Desta forma, percebe-se que a participação de todos os atores envolvidos no processo é de grande importância e podem ser determinantes no sucesso do tratamento. É imperativo buscar formas de potencializar a manutenção da medicação, com menores efeitos adversos e adesão efetiva, para tal faz se necessário que novas estratégias como formas de apresentação diferenciadas e sabor dos medicamentos, sejam também um alvo da industria na oferta de medicamentos para crianças e adolescentes.

\section{REFERÊNCIAS}

1. Brogly S, Paige W, Seage III GR, Oleske JM, Dyke RV, McIntosh K. Antiretroviral treatment in pediatric HIV infection in the United States: from clinical trials to clinical practice. JAMA 293:2213-2220, 2005.

2. D’Arminio MA, Cozzi-Lepri A, Phillips A, De Luca A, Murri R, Mussini C, Grossi P, Galli A, Zauli T, Montroni M, Tundo P. Interruption of highly active antiretroviral therapy in HIV clinical practice: results from the Italian Cohort Antiretroviral-Naïve Patients. Journal of Acquired Immune Deficiency Syndromes 38:.407-16, 2005.

3. D'Arminio MA, Cozzi-Lepri A, Rezza G, Pezzotti P, Antinori A, Phillips AN, Angarano G, Colangeli V, De Luca A, Ippolito Gd, Caggese L, Soscia F, Filice G, Gritti F, Narciso P, Tirelli U, Moroni M. Insights into the reasons for discontinuation of the first highly active therapy (HAART) regim in a cohort of antiretroviral naïve patients. I. CO.N.A. Study Group. Italian Cohort Antiretroviral-Naïve. AIDS 14:499-507, 2000.

4. Della Negra M, Marques HHS, Queiroz W, Lian YC. Manejo clínico da Aids pediátrica. Editora Atheneu, São Paulo, 1997.

5. Garcia R, Ponde M. Lack of effect of motivation on the adherence of HIV-positive/ AIDS patients to antiretroviral treatment. The Brazilian Journal of Infectious Diseases 6:494-499, 2005.

6. Grimes DA, Schhulz KF. Bias and causal associations in observational research. The Lancet 359:248-252, 2002.

7. Haddad M, Inch C, Glazier RH, Wilkins AL, Urbshott G, Bayoumi A, Rourke S Patient support and education for promoting adherence to highly active antiretroviral therapy for HIV/AIDS. Cochrane Database System Review 3:CD001442, 2000.

8. Lambert JS, Nogueira SA, Abreu TF. Manual de acompanhamento para criança infectada pelo HIV. Instituto de Puericultura e Pediatria Martagão Gesteira. Universidade Federal do Rio de Janeiro, Rio de Janeiro, 2001.

9. Ministério da Saúde. Recomendações para terapia anti-retroviral em adultos e adolescentes infectados pelo HIV. Coordenação Nacional DST e Aids. Secretaria de Vigilância em Saúde, Brasília, 2003.

10. Ministério da Saúde. Guia de tratamento clínico da infecção pelo HIV em crianças. Coordenação Nacional DST e Aids, Secretaria de Vigilância, Brasília, 2004.

11. Osterber L, Blaschke T. Adherence to medication. The New England Journal of Medicine 353:487-497, 2005.

12. Lignani LJ, Greco DB, Carneiro M. Avaliação da aderência aos anti-retrovirais em pacientes com infecção HIV/AIDS. Revista de Saúde Pública 35:495-501, 2001 .

13. Li X, Margolick JB, Conover CS, Badri S, Riddler SA, Witt MD, Jacobson LP. Interruption and discontinuation of highly active antiretroviral therapy in the multicenter AIDS cohort study. Journal of Acquired Immune Deficiency Syndromes 38: 320-328, 2005.

14. Marhefka S, Koenig LJ, Allison S, Bachanas P, Bulterys M, Bettica L, Tepper VJ, Abrams EJ. Family experiences with pediatric antiretroviral therapy: responsibilities, barries, and strategies for remembering medication. Aids Patient Care and STDs 22:637-647, 2008.

15. Medronho R, Carvalho DM, Bloch KV, Luiz RR, Werneck GL. Epidemiologia. Editora Atheneu, São Paulo, 2004. 
16. Mocroft A, Youle M, Moore A, Sabin CA, Madge S, Lepri AC, Tyrer M, Wilson D, Loveday C, Johnson Phillips AN. Reasons for modification of antiretrovirals: results from a single treatment centre AIDS 15:185-194, 2001.

17. O'brien ME, R. Clark RA, Besch CL, Myers L, Kissinger P. Patterns and correlates of discontinuation of the initial HAART regimen in an urban outpatient cohort. Journal of Acquired Immune Deficiency Syndromes 34:407-414, 2003.

18. Ridler SA, Jiang H, Tenorio A, Huang H, Kuritzkes DR, Acosta EP, Landay A, Bastow B, Haas DW, Tashima KT, Jain MK, Deeks SG, Bartlett JA. A randomized study antiviral medication at lower-versus higher-switch thresholds: AIDS Clinical Group Study A5115. Antivir Ther12:531-541, 2007.

19. Selinger-Leneman H, Matheron S, Mahamat A, Moreau J, Costtagliola D, Abgrall S. Clinical Epidemiology Group of the French Hosp Database on HIV (ANRS CO4). Dual nucleoside reverse transcriptase inhibitor therapy in the combination antiretroviral therapy era and predictors of discontinuation or switch to combination antiretroviral therapy. Journal of Acquired Immune Deficiency Syndromes 47:206-211, 2008.

20. Shibuyama S, Gevorkyan A, Yoo U, Tim S, Dzhangiryan K, Scott JD. Understanding and avoiding antiretroviral adverse events 12:1075-1090, 2006.
21. Simoni JM, Montgomery A, Martin A, New M, Demas PA, Rana S. Adherence to antiretroviral therapy for pediatric HIV infection: a qualitative systematic review with recommendations for research and clinical management. Pediatrics 119:1371-1373, 2007.

22. Van Dyke RB S, Lee, Johnson GM, Wiznia A, Mohank K, Stanley K, Morse EV, Krogstad PA, Vachnran S. Reported adherence as a determinant of response to highly active antiretrovital therapy in children who have human immunodeficiency virus infection. Pediatrics 109: 61, 2002.

23. Van Roon EN, Verzijl JM, Lenderink AW, Blans MJ, Egberts AC. Incidence of discontinuation of highly active antiretroviral combination therapy (HAART) and determinants. Journal of Acquired Immune Deficiency Syndromes 20:290-294, 1999.

24. Vreeman RC, Nyandiko VVM. A systematic review of pediatric adherence to antiretroviral therapy in low- and middle-income countries. Pediatric Infectious Disease Journal 27:686-691, 2008.

25. Wachholz NI, Ferreira J. Adherence to anriretroviral therapy in children: a study of prevalence and associated factors. Cadernos de Saúde Pública 23:424-434, 2007. 